\title{
Renal Stone
}

National Cancer Institute

\section{Source}

National Cancer Institute. Renal Stone. NCI Thesaurus. Code C26807.

Crystals in the pelvis of the kidney. 